\title{
Study on Diffusion of Low-Carbon City Technology in Guizhou Province
}

\author{
Jun Liu ${ }^{a^{*}}$, Yuanyuan Liu ${ }^{b}$, Yao Wang ${ }^{\mathrm{C}}$ \\ Guizhou University of Finance and Economics, Guiyang 550025 \\ aemail: liujunsucceed@163.com, bemail: 5267865@qq.com, cemail: 479095320@qq.com
}

\begin{abstract}
Keywords: Low-carbon City Technology; S-shaped Curve; Technology Diffusion Model
Abstract: Based on classical technology diffusion model, the diffusion model of low-carbon cities in Guizhou province is constructed, and the time path function of the technology diffusion of low carbon cities is deduced. the graphics is S-shaped curve. The results show that because of the differences of internal and external factors and the total number of "potential adopters", the time path function of low-carbon cities in different regions is different, which shows the difference between different cities to promote the technology.
\end{abstract}

\section{Introduction}

Over the past century, global climate is changing warming ${ }^{[1]}$, Human activities, especially a large amount of greenhouse gases emissions during the process of urbanization, are main factors of global climate change ${ }^{[2]}$. Climate change leads to water resources distribution imbalance, biological diversity is threatened and severe weather events frequently occur which have an adverse impact on social activities, exacerbate disease spread and threat economic development and population health $^{[3]}$.

Intergovernmental Panel on Climate Change (IPCC) studied found carbon dioxide emissions mainly come from urban areas which is about $80 \%$ of the total global carbon emissions ${ }^{[4]}$. Therefore, from the city to start, let the city development and the high carbon emissions "separate each other" is the key to achieving sustainable development

The per capita resources endowment is poor in Guizhou province and climate conditions is complex. Its karst ecological environment is particularly fragile, is more susceptible by adverse effects of climate change. In recent years, with acceleration of urbanization, the emissions of urban carbon dioxide rose year after year which has already resulted in a greater threat to ecological environment. Since 2011, Guizhou's economic growth has been higher than national average. The province will maintain rapidly economic growth and large-scale urban construction in the future ${ }^{[5]}$. City is the gathering place where population, industry and resources. It is the main carrier of economic growth ${ }^{[6]}$. High-speed economic growth led to a sharp rise in energy consumption. Rapid population gathering bring continue increasing of greenhouse gas emissions, which is a tremendous challenge to low carbon sustainable socio-economic development. Therefore, we should attach great importance to the diffusion of low-carbon technologies in the city of Guizhou province.

\section{Diffusion Model of Low-carbon City Technology in Guizhou Province}

\subsection{Basic Assumptions and Variables}

Before establish model, according to characteristics of low-carbon city technology diffusion, this paper gives following basic assumptions and related variables:

Hypothesis 1 The total number of potential adopters is $N \quad(N>0$, generally equal to the region's total population). At time $t$, there is $y(t)$ "potential adopters" has adopted low-carbon city technology, there is still $\{N-y(t)\}$ no adoption.

Hypothesis 2 The number of initial users is greater than 0; namely, at time t, $y(0)=T>0$, and $T$ is integer.

Hypothesis 3 Information is spread from external information sources, at each moment, the percentage of reaching $N-y(t)$ "non adopters" is $\alpha \times 100 \% . \alpha(\alpha<1)$ is the rate that external 
information reach to "non adopters", which is called external influence coefficient, determined by external factors.

\subsection{Time Path Functions of Low-carbon City Technology Diffusion}

\subsubsection{Time Path Function Derivation}

Starting from the point of time $t$, within the time interval $\Delta t$, the existing "non adopters" are influenced by external and internal information sources, so there are:

1) Within the time interval $\Delta t$, the number of "adopters" caused by external information sources is $\Delta y(t)^{*}=\alpha\{N-y(t)\} \Delta t$.

2) Within the time interval $\Delta t$, the number of " adopters" caused by internal information sources is $\Delta y(t)^{* *}=\beta y(t)\{N-y(t)\} \Delta t$.

If the effect that either "non adopters" were told by external information sources or internal information sources is equivalent, within the time interval $\Delta t$, the increase number of "adopters" is

$$
\Delta y(t)=\Delta y(t)^{*}+\Delta y(t)^{* *}=\{\alpha+\beta y(t)\}\{N-y(t)\} \Delta t
$$

Equation (1) is divided by $\Delta t$ on both sides, taking the limit $\Delta t \rightarrow 0$, obtain differential equation

$$
\frac{d y(t)}{d t}=\{\alpha+\beta y(t)\}\{N-y(t)\}
$$

equation (2) for the equivalent transformation, the integral is obtained

$$
y(t)=\frac{C N \exp [(\alpha+\beta N) t]-\alpha}{\beta+C \exp [(\alpha+\beta N) t]}
$$

In equation (3), $\mathrm{C}$ is constant. With the initial conditions of hypothesis $2 y(0)=T$, we can get

$$
\begin{gathered}
T=\frac{C N-\alpha}{\beta+C} \\
C=\frac{T \beta+\alpha}{N-T}
\end{gathered}
$$

Equation (5) is substituted into equation (3), the solution of differential equation(2) can be obtained.

$$
y(t)=\frac{\frac{T \beta+\alpha}{N-T} N \exp [(\alpha+\beta N) t]-\alpha}{\beta+\frac{T \beta+\alpha}{N-T} \exp [(\alpha+\beta N) t]}
$$

Due to time path function reflects the proportion change process of "adopter" accounted for "potential adopters" over time. so time path function of low-carbon city technology diffusion is

$$
Y(t)=\frac{1}{N} y(t)=\frac{1}{N} \frac{\frac{T \beta+\alpha}{N-T} N \exp [(\alpha+\beta N) t]-\alpha}{\beta+\frac{T \beta+\alpha}{N-T} \exp [(\alpha+\beta N) t]}
$$




\subsubsection{Curve of Time Path Function}

Curve diagram of time path function is drawn by using function graph. First, in order to find the inflection point of time path function, we get the first order derivative and the second order derivative of $Y(t)$. then in order to obtain the concave convex interval of time path function, we get the third derivative of $Y(t)$.

Since equation (7) is an elementary function, it must be continuous, so its first order derivative is obtained.

$$
Y^{\prime}(t)=\frac{1}{N}\left\{\frac{T \beta+\alpha}{N-T} \exp [(\alpha+\beta N) t](\alpha+\beta N)^{2}\right\}\left\{\beta+\frac{T \beta+\alpha}{N-T} \exp [(\alpha+\beta N) t]\right\}^{-2}
$$

In the same way, the first derivative of equation (8) is obtained.

$$
Y^{\prime \prime}(t)=\left\{(\alpha+\beta N)-2 \frac{C(\alpha+\beta N) \exp [(\alpha+\beta N) t]}{\beta+C \exp [(\alpha+\beta N) t]}\right\} Y^{\prime}(t)
$$

Equation (8) is substituted into equation (9).

$$
Y^{\prime \prime}(t)=\left\{(\alpha+\beta N)-\frac{2 C(\alpha+\beta N) \exp [(\alpha+\beta N) t]\}\left\{\frac{C}{\beta} \exp [(\alpha+\beta N) t](\alpha+\beta N)^{2}\right\}\{\beta+C \exp [(\alpha+\beta N) t]}{\beta}[(\alpha+\beta N) t]\right\}^{-2}
$$

Order $Y^{\prime \prime}(t)=0$, we get the inflection point of equation (7). From hypothesis 2, 3, 4, we know $Y^{\prime}(t)>0$, so $Y^{\prime \prime}(t)=0$ is equivalent to

$$
(\alpha+\beta N)-2 \frac{C(\alpha+\beta N) \exp [(\alpha+\beta N) t]}{\beta+C \exp [(\alpha+\beta N) t]}=0
$$

The solution of equation (11) is $t=t_{\text {inf lexion }}=\frac{\ln \frac{\beta}{C}}{(\alpha+\beta N)}$, and $\left(t_{\text {inf lexion }}, Y\left(t_{\text {inf lexion }}\right)\right)$ is the inflection point of equation (7).

With inflection point of equation (7), we can get concave \& convex interval. Equation (10) is an elementary function, which first order derivative is

$$
Y^{\prime \prime \prime}(t)=\phi^{4} C \exp (\phi t)[\beta+C \exp (\phi t)]^{-3}\left\{\beta-2 C \exp (\phi t)-3 C \exp (\phi t)[\beta-C \exp (\phi t)] \cdot[\beta+C \exp (\phi t)]^{-1}\right\} \frac{1}{N}
$$

According to definition of concave \& convex function, $Y^{\prime \prime}(0)=\left(\frac{\alpha \beta+\beta^{2} N}{\beta+C}\right)\left(\frac{C(\alpha+\beta N)^{2}}{(\beta+C)^{2}}\right)>0$, and in the interval $t \in\left[0, t_{\text {inf lexion }}\right), Y^{\prime \prime \prime}(t)>0$, namely, $Y^{\prime \prime}(t)$ is monotone increasing in the interval $t \in\left[0, t_{\text {inf lexion }}\right)$. So $Y^{\prime \prime}(t)>0$ equation (7) is concave in the interval $t \in\left[0, t_{\text {inf lexion }}\right)$. In the interval $t \in\left(t_{\text {inf lexion }},+\infty\right), Y^{\prime \prime \prime}(t)<0$, namely, $Y^{\prime \prime}(t)$ is monotone decreasing in the interval $t \in\left(t_{\text {inflexion }},+\infty\right)$. So $Y^{\prime \prime}(t)<0$, equation (7) is convex in the interval $t \in\left(t_{\text {inf lexion }},+\infty\right)$.

In summary, equation (7) graphics can be made as shown in Figure 1, which is a S shaped curve. The proportion of "adopted" accounted for "potential adopters" is decided by four parameters 
$\alpha, \beta, N, T$. The change is reflected in the graphics, that is, the diffusion of the low carbon city technology has a $\mathrm{S}$ - shaped growth pattern over time.

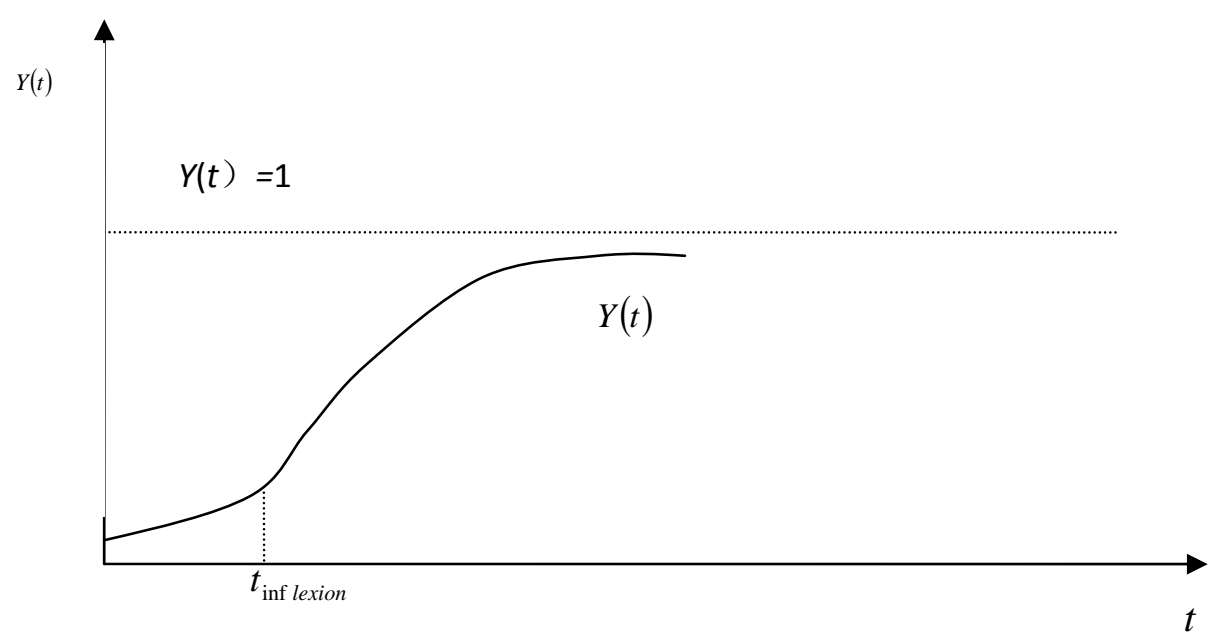

Fig. 1 Time Path Function Curves of Technology Diffusion in Low-carbon Cities

\section{Conclusion}

First, the difference of external factors in different regions lead to different parameter $\alpha$. Second, the difference of internal factors in different regions lead to different parameter $\beta$. In addition, the total number of potential adopters $N$ and the number of initially users $T$ in different regions is generally different. Therefore, the difference of parameters $\alpha, \beta, N$ and $T$ in different regions lead to the difference of time path function. At the same time, the difference of time path function values show the difference between the two regions.

As time goes on, this difference will be gradually expansion, stability, and gradually narrow. The evolution of this three stage has a universal law. Therefore, the conclusion of this paper can be used to explain the difference of low-carbon cities technology diffusion between different regions.

\section{Acknowledgements}

Research on evaluation of tobacco farmers classification and differential management in southwestern Guizhou (2011-15); Study on the mechanism of foreign assets of tobacco farmers cooperatives inclusive (2015-9); Guizhou Province Science and Technology Fund project ( Qiankehe J [2015] No. 2028); Science and technology project of China National Tobacco Corp Guizhou Branch (201224); National social science fund project (14CJL032)

\section{Reference}

[1] World Resources Institute. Climate analysis indicators tool[R]. New York , U. S. A. : WRI,2010:3-4.

[2] Malla S. CO2 emissions from electricity generation in seven Asia-Pacific and North American countries: A decomposition analysis[J].Energy Policy, 2009, (37):1-9.

[3] Blanford G. J., Richels R. G., Rutherford T. F.Revised emissions growth projections for China: why post-kyoto climate policy must look east[R] USA: Harvard Project on International Climate Agreements, 2008: 2-6. 
[4] Pachauri R. K., Reisinger A. Climate change 2007: synthesis report[R]. Geneva , Switzerland : Intergovernmental Panel on Climate Change,2007:1-5.

[5] Intergovernmental Panel on Climate Change. 2006 IPCC guidelines for national greenhouse gas inventories[M]. Japan: the Institute for Global Environmental Strategies (IGES), 2006.

[6] Liang Dan. Progress in research on technology diffusion [J]. research management, 2005, 26(4): 29-34. 